\title{
Cerebellar ataxia with sensory ganglionopathy; does autoimmunity have a role to play?
}

Panagiotis Zis $^{1,2^{*}}$ D, Ptolemaios Georgios Sarrigiannis ${ }^{1}$, Dasappaiah Ganesh Rao ${ }^{1}$, Nigel Hoggard ${ }^{3}$, David Surendran Sanders ${ }^{2,4}$ and Marios Hadjivassiliou ${ }^{1,2}$

\begin{abstract}
Background and purpose: Cerebellar ataxia with sensory ganglionopathy (SG) is a disabling combination of neurological dysfunction usually seen as part of some hereditary ataxias. However, patients may present with this combination without a genetic cause.

Methods: We reviewed records of all patients that have been referred to the Sheffield Ataxia Centre who had neurophysiological and imaging data suggestive of SG and cerebellar ataxia respectively. We excluded patients with Friedreich's ataxia, a common cause of this combination. All patients were screened for genetic causes and underwent extensive investigations.

Results: We identified 40 patients (45\% males, mean age at symptom onset $53.7 \pm 14.7$ years) with combined cerebellar ataxia and SG. The majority of patients (40\%) were initially diagnosed with cerebellar dysfunction and 30\% were initially diagnosed with SG. For 30\% the two diagnoses were made at the same time. The mean latency between the two diagnoses was $6.5 \pm 8.9$ years (range $0-44$ ). The commonest initial manifestation was unsteadiness (77.5\%) followed by patchy sensory loss (17.5\%) and peripheral neuropathic pain (5\%).

Nineteen patients (47.5\%) had gluten sensitivity, of whom 3 patients (7.5\%) had biopsy proven coeliac disease. Other abnormal immunological tests were present in another 15 patients. Six patients had malignancy, which was diagnosed within 5 years of the neurological symptoms. Only 3 patients (7.5\%) were classified as having a truly idiopathic combination of cerebellar ataxia with SG.
\end{abstract}

Conclusion: Our case series highlights that amongst patients with the unusual combination of cerebellar ataxia and SG, immune pathogenesis plays a significant role.

Keywords: Ganglionopathy, Cerebellar ataxia, Gluten, Autoimmunity

\section{Introduction}

Ataxia is a term used to describe unsteadiness and poor co-ordination of movements. It can occur secondary to cerebellar dysfunction (cerebellar ataxia) or because of dysfunction within the peripheral or central sensory pathways (sensory ataxia).

\footnotetext{
* Correspondence: takiszis@gmail.com

${ }^{1}$ Academic Department of Neurosciences, Sheffield Teaching Hospitals NHS Foundation Trust, Sheffield, UK

${ }^{2}$ University of Sheffield, Sheffield, UK

Full list of author information is available at the end of the article
}

Cerebellar ataxia can be inherited (e.g. Friedreich's ataxia and spinocerenellar ataxias, known as SCAs) or acquired (e.g. gluten ataxia and paraneoplastic) [1].

Sensory ataxia is commonly seen in neuropathies that involve sensory fibers and are more prominent in pure sensory neuropathies involving the dorsal root ganglia, commonly referred to as sensory ganglionopathies (SG) [2]. Causes of SG can be inherited (e.g. mitochondrial disorders) or acquired (e.g. paraneoplastic) [3-5].

Cerebellar ataxia combined with SG is a relatively rare neurological combination, which can sometimes be seen in the context of hereditary ataxias [2] (e.g. 
Friedreich's ataxia and SCA4, mitochondrial disease and Cerebellar ataxia, neuropathy and vestibular areflexia syndrome (CANVAS) [6]) or as a result of exposure to toxins (e.g. amiodarone [7]).

We present a case series of patients with this unusual combination, in an attempt to shed light into possible underlying aetiology.

\section{Methods}

\section{Standard protocol approvals, registrations, and patient} consents

This is a retrospective observational case series of patients regularly attending the Ataxia clinic based at the Royal Hallamshire Hospital (Sheffield, UK). The South Yorkshire Research Ethics Committee has confirmed that no ethical approval is indicated given that all investigations were clinically indicated and did not form part of a research study.

\section{Patient selection}

All recruited patients had clinical and neuroimaging evidence of progressive cerebellar dysfunction as well as clinical and neurophysiological evidence of SG.

\section{Neurophysiological assessments}

All patients underwent detailed nerve conduction studies including median (sensory and motor), ulnar (sensory and motor), superficial radial (sensory), sural (sensory), superficial peroneal (sensory) and tibial (motor). All sensory nerve conduction studies were performed bilaterally. On all occasions supramaximal stimulation of the nerves was performed.

The diagnosis of SG was based on the established diagnostic criteria [8,9]. Clinically patients that presented with signs and symptoms of patchy sensory symptoms and reduced or absent tendon reflexes underwent nerve conduction studies. SG was confirmed electrophysiologically with complete absence of sensory nerve action potentials (SNAPs) or asymmetrical sensory fiber involvement (asymmetrical SNAPs) with no motor involvement.

\section{Neuroimaging assessments}

All patients underwent a magnetic resonance imaging (MRI) of the brain and a magnetic resonance spectroscopy (MRS) of the cerebellum. The latter technique is validated and is used to determine the presence of cerebellar dysfunction, even in the absence of cerebellar atrophy [10]. We measured the $\mathrm{N}$-acetyl-aspartate/creatine (NAA/Cr) ratios in the vermis and the cerebellar hemispheres. Only patients with cerebellar atrophy and/or abnormal NAA/Cr ratios in either the vermis or the hemispheres and/or cerebellar atrophy in the MRI where considered as having cerebellar dysfunction.

\section{Serologic testing}

Serological testing was performed and included extensive testing for possible acquired causes of cerebellar ataxia and sensory ganglionopathy. Human leukocyte antigen (HLA) typing was performed by the regional blood-transfusion service.

\section{Genetic testing}

Patients with early onset cerebellar ataxia and/or family history of ataxia were tested genetically for possible genetic causes. A muscle biopsy was done in patients with a suspected mitochondrial disease. By default we did not include in this case series patients with Friedreich's ataxia (FA) as this is a well known cause of this combination of neurological deficits.

\section{Enteropathy}

Patients with gluten sensitivity underwent gastroscopy and duodenal biopsy to establish the presence of enteropathy. All biopsies were histologically assessed for evidence of enteropathy (triad of villous atrophy, crypt hyperplasia, and increase in intraepithelial lymphocytes).

\section{Statistical analysis}

A database was developed using the statistical software package SPSS (version 23.0 for Macintosh). Descriptive statistics were examined for each variable.

\section{Results}

\section{Clinical characteristics}

We identified 40 patients (45\% males) with combined cerebellar ataxia and sensory ganglionopathy. Mean age at onset of the symptoms was $53.7 \pm 14.7$ years (range $8-80$ ) and mean age at first presentation was $56.6 \pm$ 13.5 years (range 14-81). At presentation, the majority of patients $(40 \%)$ were diagnosed with cerebellar ataxia and $30 \%$ were diagnosed with SG. For 30\% the two diagnoses were made at the same time. The mean latency between the two diagnoses was $6.5 \pm 8.9$ years (range 0 44). The commonest initial manifestation was unsteadiness $(77.5 \%)$ followed by patchy sensory loss $(17.5 \%)$ and peripheral neuropathic pain (5\%).

\section{Neurophysiology}

Sixteen patients (40\%) had absent SNAPs, whereas the rest had asymmetrical SNAPs. No patients had abnormal compound muscle action potentials (CMAPs). Table 1 summarizes the neurophysiological characteristics of a typical patient with asymmetrical SNAPs (Patient 1) and 


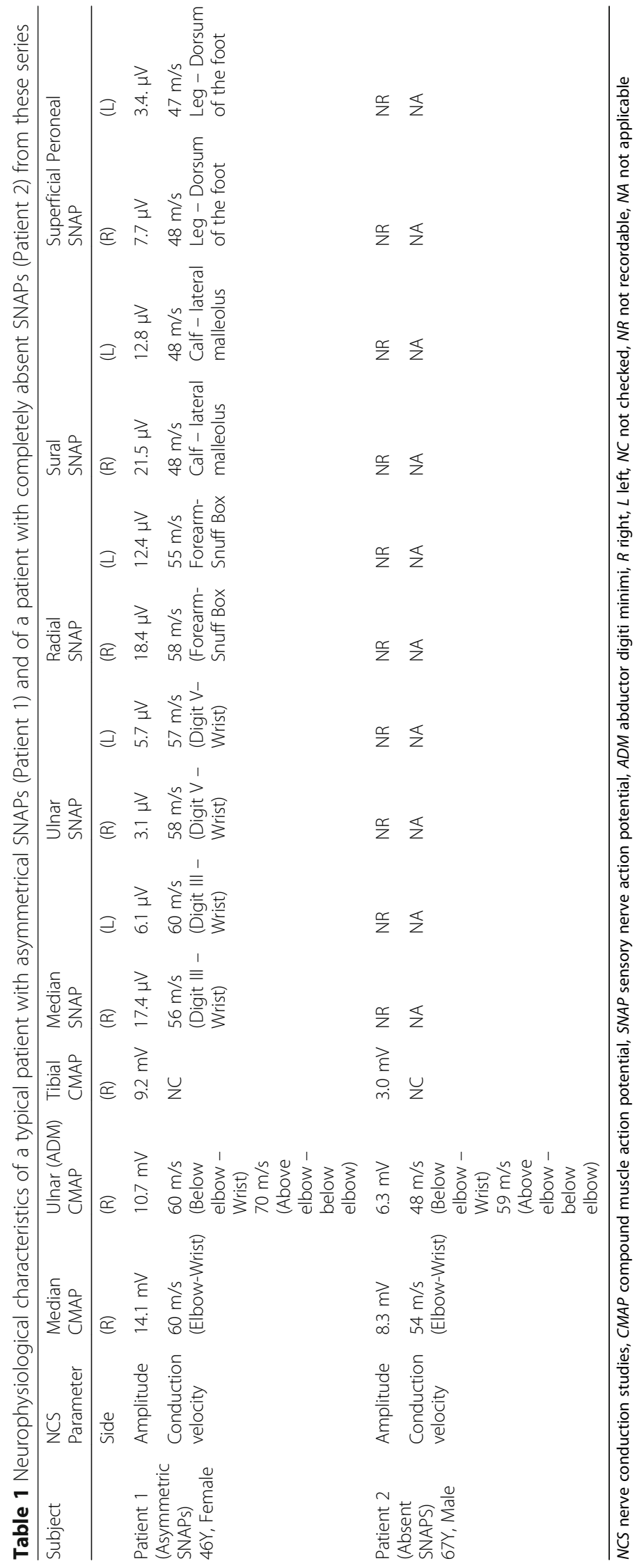


of a patient with completely absent SNAPs (Patient 2) from these series.

\section{Neuroimaging characteristics}

All patients had abnormal MRS at diagnosis. Fifteen patients $(37.5 \%)$ had low $\mathrm{NAA} / \mathrm{Cr}$ ratio only in the vermis $(<0.96)$ and 6 patients $(15 \%)$ had low NAA/Cr ratio only in the hemispheres $(<1)$. Nineteen patients (47.5\%) had low NAA/Cr ratio in both the vermis and the hemispheres. Only $55 \%$ of the patients had cerebellar atrophy in the initial MRI.

\section{Evidence of autoimmunity}

Nineteen patients $(47.5 \%)$ had gluten sensitivity, of whom 3 patients $(7.5 \%)$ had biopsy proven coeliac disease. Other abnormal immunological tests were present in another 15 patients (i.e. GAD, ANA etc.), of whom 4 patients (10\%) had biopsy proven Sjogren's syndrome.

\section{Cancer}

Nine patients had a history or developed malignancy (3 bowel, 1 melanoma, 1 light chain myeloma, 2 ovarian, 1 uterus and 1 of unknown primary location). However, only in 6 patients of them the malignancy was diagnosed within 5 years of the neurological symptoms. Anti-Yo antibodies were found in one patient.

\section{Genetic causes}

Nineteen patients were investigated for familial ataxias using next generation sequencing ataxia panel containing 42 genes. In selected patients genetic testing for common mitochondrial mutations (including POLG1) and muscle biopsies were performed. For two of them a genetic cause was identified (one mitochondrial, one SCA18).

\section{HLA type}

HLA typing was performed in 34 patients. Of them, 18 patients $(52.9 \%)$ had the DQ2 type, 6 patients (17.6\%) had the DQ8 type, 9 patients (26.5\%) had the DQ1 type and 1 patient $(2.9 \%)$ had another HLA type.

\section{Truly idiopathic cases}

After extensive immunological and genetic screening, only 3 patients $(7.5 \%)$ were classified as having a truly idiopathic combination of cerebellar ataxia with SG (Table 2).

\section{Discussion}

Our case series highlights that amongst patients with cerebellar ataxia and sensory neuronopathy, where a genetic or a malignant cause is not identified, immune pathogenesis is a likely cause. In fact out of 40 patients, only 2 were found to have a genetic cause and only 6 patients had malignancy, which was diagnosed within 5 years of their symptoms. From the remaining 32 patients the vast majority (91\%) had evidence of autoimmunity and only 3 patients (9\%) were classified as truly idiopathic.

Whether considering the cut-off of 5 years for a neurological syndrome to be classified as paraneoplastic is a matter of debate [11]. This time period has been based on reports showing that in the majority of cases the interval between the paraneoplastic neurological syndrome and the diagnosis of malignancy is less than 5 years [11]. However in our case series, there are patients who exceed this interval. The type of malignancy, however, may influence this interval.

The HLA type has been linked to predisposition to autoimmunity. In our case series $70.5 \%$ of patients had the DQ2 or the DQ8 HLA subtypes, which are known to be associated with autoimmunity. This percentage is significantly higher compared to the $40 \%$ of the general population [12]. In addition, in our cohort, evidence for autoimmunity (serological evidence of gluten sensitivity and/or positive other antibody titers) was present in $90 \%$ of the patients.

Our findings should be interpreted with some caution given the limitations of our design. Firstly, as this is a retrospective observational case series of patients regularly attending our Ataxia clinic not all patient's had full genetic screening, which means that the percentage of genetic causes might be higher than the one we reported. However, the bias is probably minimized by the fact that we genetically tested patients with early onset and/or family history of ataxia and SG. When clinically suspected, patients also underwent genetic testing for mitochondrial diseases including muscle biopsies. Despite this it is possible that in some patients mitochondrial aetiology $[13,14]$ may have been missed. Secondly, we haven't routinely been examining for the vestibulo-ocular reflex and therefore cases of CANVAS may have been missed. However, our understanding is that most cases with CANVAS, unlike the series here, tend to have a family history.

Both cerebellar ataxia (gluten ataxia) and sensory ganglionopathy in isolation have been previously linked to gluten sensitivity $[4,10,15]$. Almost half of the patients in this report $(47.5 \%)$ had serological evidence of gluten sensitivity without enteropathy (coeliac disease). Gluten free diet has already been shown to be beneficial in such patients [4] and should be considered in all patients with cerebellar ataxia and sensory ganglionopathy in the presence of serological markers of sensitivity to gluten. 


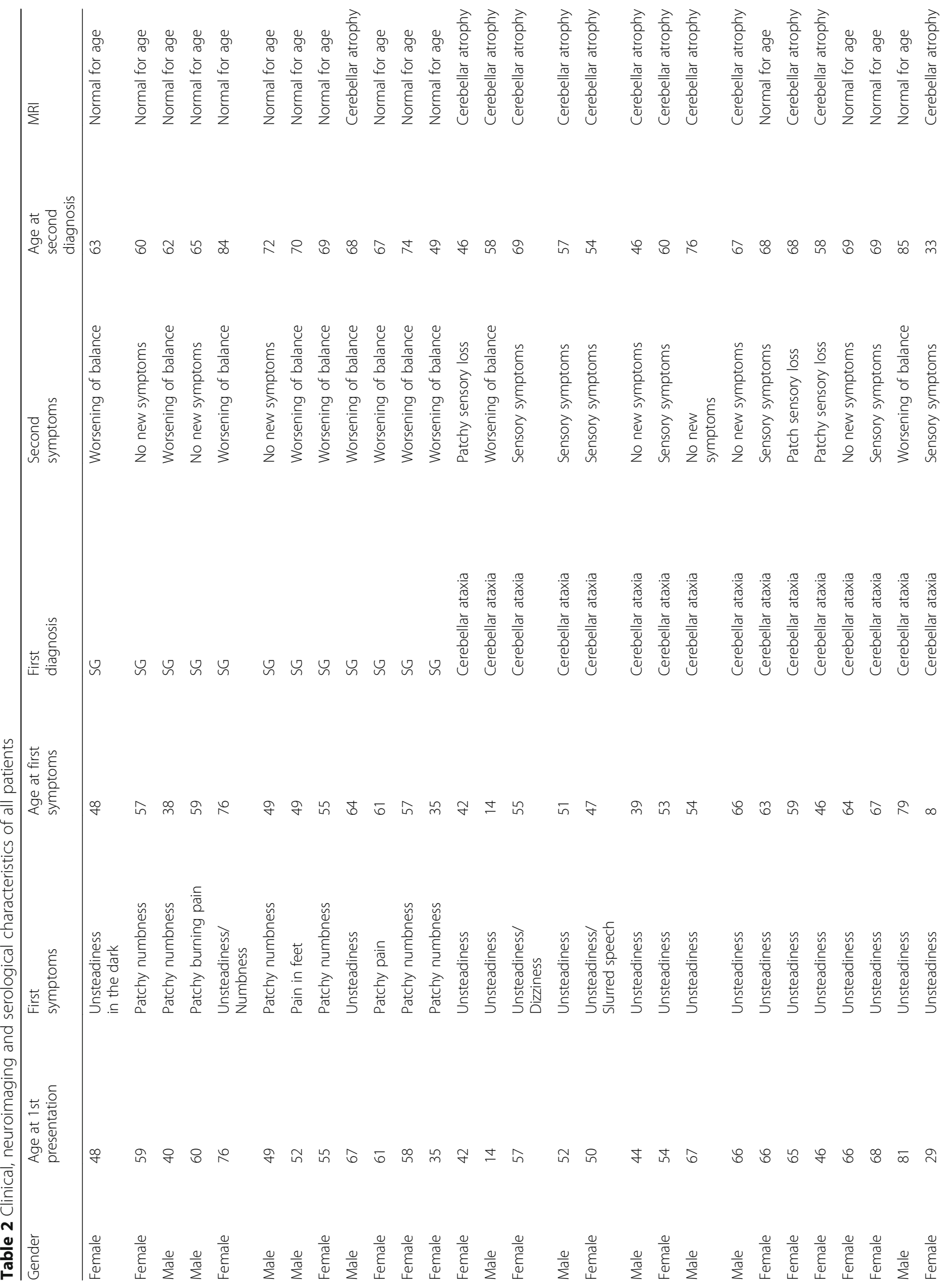




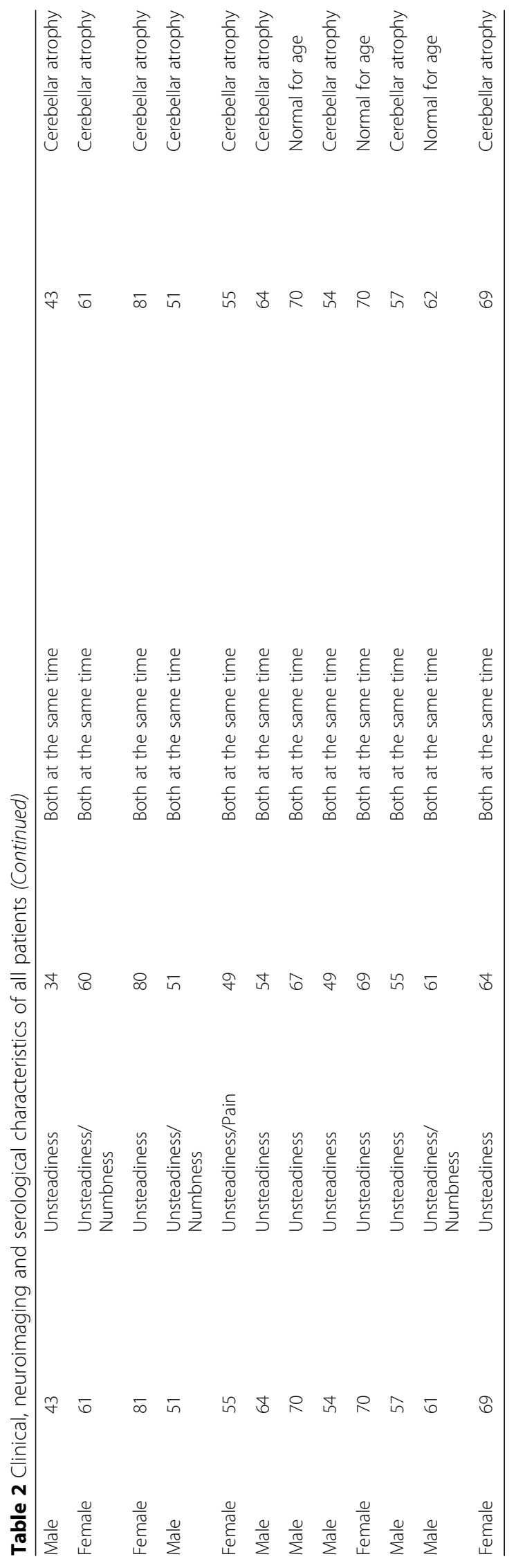




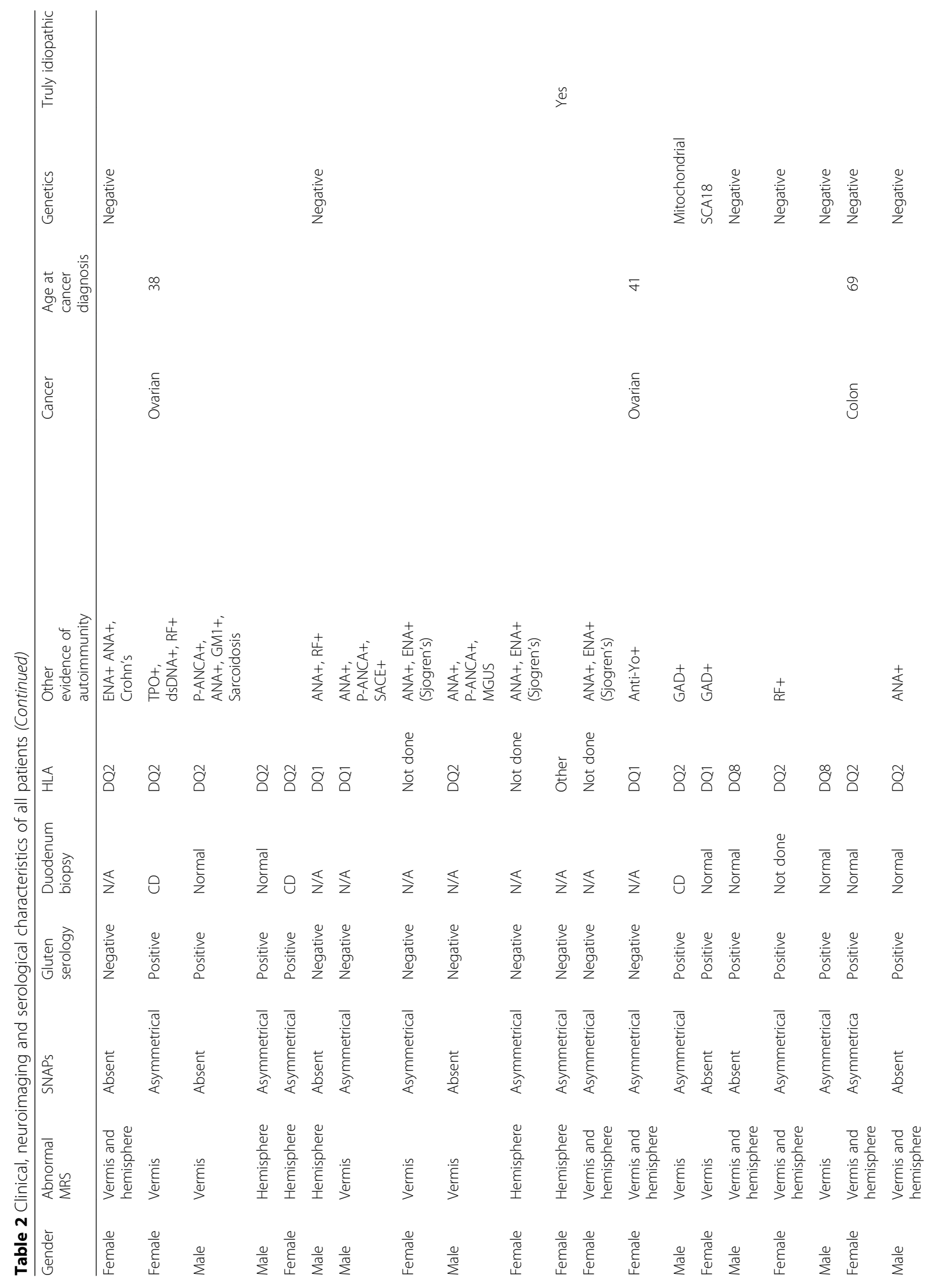




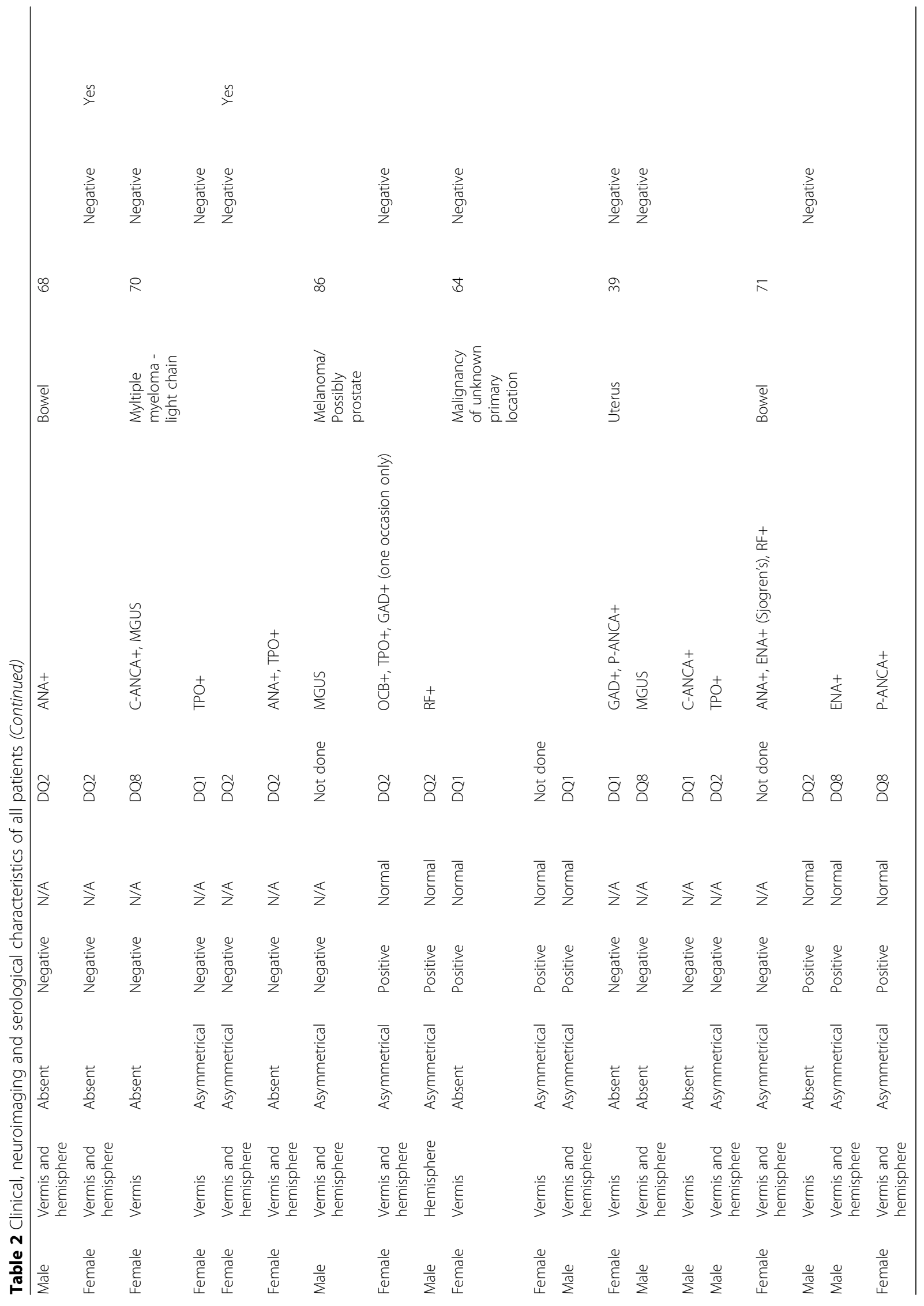




\section{Acknowledgements}

None.

\section{Funding}

This is a summary of independent research supported by NIHR Sheffield Biomedical Research Centre (Translational Neuroscience). The views expressed are those of the author(s) and not necessarily those of the NIHR Sheffield Biomedical Research Centre, NHS, the NIHR or the Department of Health.

\section{Availability of data and materials}

The datasets analyzed during the current study are available from the corresponding author on reasonable request.

\section{Authors' contributions}

PZ: drafting/revising the manuscript, study concept and design, data collection, statistical analysis, accepts responsibility for conduct of research and final approval. MH: drafting/revising the manuscript, study concept and design, data collection, accepts responsibility for conduct of research and final approval. PGS, DGR, NH and DS: drafting/revising the manuscript, data collection, accept responsibility for conduct of research and final approval.

\section{Ethics approval and consent to participate}

The South Yorkshire Research Ethics Committee has confirmed that no ethical approval is indicated given that all investigations were clinically indicated and did not form part of a research study.

\section{Consent for publication}

Not applicable.

\section{Competing interests}

The authors declare that they have no competing interests.

\section{Publisher's Note}

Springer Nature remains neutral with regard to jurisdictional claims in published maps and institutional affiliations.

\section{Author details}

${ }^{1}$ Academic Department of Neurosciences, Sheffield Teaching Hospitals NHS Foundation Trust, Sheffield, UK. ${ }^{2}$ University of Sheffield, Sheffield, UK. ${ }^{3}$ Department of Neuroradiology, Sheffield Teaching Hospitals NHS Foundaiton Trust, Sheffield, UK. ${ }^{4}$ Academic Unit of Gastroenterology, Sheffield Teaching Hospitals NHS Foundation Trust, Sheffield, UK.

\section{Received: 7 November 2017 Accepted: 8 December 2017}

Published online: 22 December 2017

\section{References}

1. Ramirez-Zamora A, Zeigler W, Desai N, Biller J. Treatable causes of cerebellar ataxia. Mov Disord. 2015;30:614-23.

2. Sghirlanzoni A, Pareyson D, Lauria G. Sensory neuron diseases. Lancet Neurol. 2005;4:349-61.

3. Zis P, Sarrigiannis PG, Rao DG, Hewamadduma C, Hadjivassiliou M. Chronic idiopathic axonal polyneuropathy: a systematic review. J Neurol. 2016; 263(10):1903-10.

4. Hadjivassiliou M, Rao DG, Grinewald RA, Aeschlimann DP, Sarrigiannis PG, Hoggard N, et al. Neurological dysfunction in coeliac disease and noncoeliac gluten sensitivity. Am J Gastroenterol. 2016;111(4):561-7.

5. Scaravilli F, An SF, Groves M, Thom M. The neuropathology of paraneoplastic syndromes. Brain Pathol. 1999;9:251-60.

6. Szmulewicz DJ, McLean CA, MacDougall HG, Roberts L, Storey E, Halmagyi GMCANVAS. An update: clinical presentation, investigation and management. J Vestib Res. 2014;24(5-6):465-74. https://doi.org/10.3233/NES140536.

7. Hilleman D, Miller MA, Parker R, Doering P, Pieper JA. Optimal management of amiodarone therapy: efficacy and side effects. Pharmacotherapy. 1998;18: 138s-45s. https://doi.org/10.1002/j.1875-9114.1998.tb03950.x.

8. Camdessanché JP, Jousserand G, Ferraud K, Vial C, Petiot P, Honnorat J, Antoine JC. The pattern and diagnostic criteria of sensory neuronopathy: a case-control study. Brain 2009 Jul;132(Pt 7):1723-1733. doi: https://doi.org/ 10.1093/brain/awp136. Epub 2009 Jun 8.
9. Zis P, Hadjivassiliou M, Sarrigiannis PG, Barker SJEA, Rao DG. Rapid neurophysiological screening for sensory ganglionopathy: a novel approach. Brain Behav. 2017; https://doi.org/10.1002/brb3.880.

10. Hadjivassiliou M, Wallis LI, Hoggard N, Grünewald RA, Griffiths PD, Wilkinson IDMR. Spectroscopy and atrophy in gluten, Friedreich's and SCA6 ataxias. Acta Neurol Scand. 2012 Aug 1;126(2):138-43.

11. Zis P, Rao DG, Wagner BE, Nicholson-Goult L, Hoggard N, Hadjivassiliou M Cerebellar ataxia and sensory ganglionopathy associated with light-chain myeloma. Cerebellum Ataxias. 2017 Jan 5;4:1.

12. Megiorni F, Mora B, Bonamico M, Barbato M, Nenna R, Maiella G, Lulli P, Mazzilli MC. HLA-DQ and risk gradient for celiac disease. Hum Immunol. 2009 Jan 31;70(1):55-9.

13. Mignarri A, Cenciarelli S, Da Pozzo P, Cardaioli E, Malandrini A, Federico A, Dotti MT. Mitochondrial recessive ataxia syndrome: a neurological rarity not to be missed. J Neurol Sci. 2015 Feb 15;349(1-2):254-5.

14. Bargiela D, Shanmugarajah P, Lo C, Blakely EL, Taylor RW, Horvath R, Wharton S, Chinnery PF, Hadjivassiliou M. Mitochondrial pathology in progressive cerebellar ataxia. Cerebellum Ataxias. 2015 Dec 4;2:16. https:// doi.org/10.1186/s40673-015-0035-x. eCollection 2015.

15. Zis $P$, Rao DG, Sarrigiannis PG, Aeschlimann P, Aeschlimann DP, Sanders D, Grünewald RA, Hadjivassiliou M. Transglutaminase 6 antibodies in gluten neuropathy. Dig Liver Dis. 2017 Nov:49(11):1196-200.

\section{Submit your next manuscript to BioMed Central and we will help you at every step:}

- We accept pre-submission inquiries

- Our selector tool helps you to find the most relevant journal

- We provide round the clock customer support

- Convenient online submission

- Thorough peer review

- Inclusion in PubMed and all major indexing services

- Maximum visibility for your research

Submit your manuscript at www.biomedcentral.com/submit
Ciomed Central 